\title{
Angiotomografía en sospecha de enfermedad oclusiva aorto-ilíaca (o síndrome de Leriche)
}

CT angiography for suspected aortoiliac occlusive disease (or Leriche syndrome)

Angiotomografia por suspeita de doença oclusiva aorto-ilíaca (ou síndrome de Leriche)

Yelson Alejandro Picón-Jaimes, MD., Esp., MSc. ${ }^{1} \mathbb{D}$, Jonathan Javier Díaz-Jurado, Int. $^{2}$ (D) Javier Esteban Orozco-Chinome, MD. MSc. ${ }^{3}$ (D), Paola Alexandra RamírezRodríguez, Est. ${ }^{4}$ (D), Natalia Andrea Arciniegas-Torres, Est. ${ }^{4}$ (D), María Alejandra Hernández-Sarmiento, Est. ${ }^{4}$ (D) Sergio Alberto Villabona-Rosales, MD. Esp. ${ }^{5}$ (D)

1. Médico, Especialista en Bioética aplicada al campo clínico experimental, Magíster en Bioética. Centro Médico Medicien-Servicios Médicos Dial SA. Santiago, Chile.

2. Médico Interno. Universidad Autónoma de Bucaramanga, Bucaramanga, Santander, Colombia.

3. Médico. Diploma en Dermatología. Magíster en Medicina Estética, Regenerativa y Antienvejecimiento. Asociación Chilena de Seguridad ACHS. Santiago, Chile.

4. Estudiante de Medicina. Universidad Autónoma de Bucaramanga, Bucaramanga, Santander, Colombia.

5. Médico. Especialista en Medicina de Urgencias. Fundación oftalmológica de Santander-Clínica Carlos Ardila Lulle (FOSCAL). Universidad Autónoma de Bucaramanga, Bucaramanga, Santander, Colombia.

Correspondencia. Yelson Alejandro Picón Jaimes. Centro Médico Medicien-Servicios Médicos Dial SA. Dirección. Av Pocuro 2260, Providencia, Santiago, Chile. Teléfono. +56 948991158 Email. ypicon@unab.edu.co

\section{INFORMACIÓN DEL ARTÍCULO:}

Artículo recibido: 02 de octubre 2019

Artículo aceptado: 30 de abril 2020

Doi: https://doi.org/10.29375/01237047.3732

Cómo citar: Picón-Jaimes YA, Díaz-Jurado JJ, Orozco-Chinome JE, RamírezRodríguez PA, Arciniegas-Torres NA, Hernández-Sarmiento MA, Villabona-Rosales SA. Angiotomografia en sospecha de enfermedad oclusiva aorto-ilíaca (o síndrome de Leriche). MedUNAB. 2020;23(2): 301-306. doi: 10.29375/01237047.3732

\section{RESUMEN}

Introducción. El síndrome de Leriche fue definido en 1940 por René Leriche como una enfermedad oclusiva aorto-ilíaca. Su importancia radica en que ocupa el segundo lugar en incidencia dentro de las enfermedades oclusivas arteriales, 
después de la enfermedad de la arteria femoral superficial. Objetivo. Presentar el caso clínico de un paciente con Síndrome de Leriche cuyo diagnóstico trombótico fue confirmado por una angiotomografía computarizada con su respectiva reestructuración en 3D. Adicionalmente, se informa al lector sobre los datos propios de la patología a través de una breve revisión de la literatura. Caso clínico. Paciente masculino con síntomas clínicos agudos de enfermedad oclusiva aortoilíaca o síndrome de Leriche en una extremidad. Se realiza el respectivo procesamiento de imagen asistida por computador (reconstrucción tridimensional) que evidencia la patología a pesar de que la ecografía Doppler no determinó trombosis inicial. Discusión. En procesos oclusivos arteriales se emplean técnicas no invasivas como la ecografía y la angiotomografía. Sin embargo, esta última es la técnica por excelencia, sobre todo para procesos trombóticos, pues agiliza la anticoagulación, así como el abordaje terapéutico. Conclusiones. La angiotomografía es una técnica no invasiva con alta sensibilidad y especificidad para detectar estenosis aorto-ilíaca. Se ha convertido en una gran herramienta diagnostica por sus alcances imagenológicos, como la obtención de imágenes iso volumétricas, que permiten evaluar todo el trayecto arterial en los diferentes planos, por medio del uso de medios de contraste, superando en resultados a la ecografía.

Palabras claves:

Síndrome de Leriche; Aorta Abdominal; Enfermedades de la Aorta; Arteria Ilíaca;Claudicación Intermitente.

\section{ABSTRACT}

Introduction. Leriche syndrome was defined in 1940 by René Leriche as an aortoiliac occlusive disease. Its importance lies in it occupying second place in the incidence of occlusive arterial diseases, after superficial femoral artery disease. Objective. Present the clinical case of a patient with Leriche syndrome whose diagnosis of thrombosis was confirmed by a computed tomography angiography with its respective 3D reconstruction. Additionally, the reader is given information about the pathology through a brief summary of the literature. Clinical case. Male patient with acute clinical symptoms of aortoiliac occlusive disease or Leriche syndrome in one limb. The respective computer-assisted image processing (three-dimensional reconstruction) is carried out, which shows the pathology, despite the Doppler ultrasound not initially establishing thrombosis. Discussion. Non-invasive techniques are used in procedures for occlusive arteries, such as ultrasound and CT angiography. However, the latter is a technique par excellence, above all for thrombosis procedures, as well as the therapeutic approach. Conclusions. CT angiography is a non-invasive technique with high sensitivity and specificity in the detection of aortoiliac stenosis. It has become a great diagnostic tool because of its imagery scope, such as obtaining isovolumic images, which enable the assessment of the entire arterial route in different planes, through the use of contrast media, producing more results than the ultrasound.

Keywords:

Leriche Syndrome; Abdominal Aorta; Aortic Diseases; Iliac Artery; Intermittent Claudication.

\section{RESUMO}

Introdução. A síndrome de Leriche foi definida em 1940 por René Leriche como uma doença oclusiva aorto-ilíaca. Sua importância reside no fato de ocupar o segundo lugar em incidência dentro das doenças arteriais obstrutivas, após a doença na artéria femoral superficial. Objetivo. Apresentar o caso clínico de um paciente com síndrome de Leriche cujo diagnóstico trombótico foi confirmado por uma angiotomografia computadorizada (angio-TC) com reestruturação em 3D. Adicionalmente, o leitor é informado sobre os dados próprios da patologia através de uma breve revisão de literatura. Caso clínico. Paciente de sexo masculino com sintomas clínicos agudos da doença oclusiva aortoilíaca ou síndrome de Leriche em uma extremidade. Foi realizado o processamento da imagem assistida por computador (reconstrução tridimensional), evidenciando a patologia, ainda que a ultrassonografia Doppler não determinou trombose inicial. Discussão. Em processos oclusivos arteriais são utilizadas técnicas não invasivas como a ultrassonografia e a angiotomografia. No entanto, a angiotomografia é a técnica padrão ouro, principalmente para processos trombóticos, pois 
acelera a anticoagulação e a abordagem terapêutica. Conclusão. A angiotomografia é uma técnica não invasiva com alta sensibilidade e especificidade para detectar estenose aorto-ilíaca. Tornou-se uma ótima ferramenta de diagnóstico por suas características imagenológicas, como a obtenção de imagens isovolumétricas que permitem avaliar todo o trajeto arterial nos diferentes planos, através do uso de meios de contraste, superando os resultados da ultrassonografia.

Palavras-chave:

Síndrome de Leriche; Aorta Abdominal; Doenças da Aorta; Artéria Ilíaca; Claudicação Intermitente.

\section{Introducción}

El síndrome de Leriche fue definido en 1940 por René Leriche como una Enfermedad Oclusiva Aorto-Ilíaca (EAOI), la cual ocupa el segundo lugar en incidencia entre las enfermedades oclusivas arteriales, después de la enfermedad de la arteria femoral superficial (1-2). La incidencia y prevalencia exacta de esta enfermedad son difíciles de determinar debido a que muchos pacientes que tienen esta patología son asintomáticos. Los casos que se registran en la literatura han sido obtenidos gracias al uso de mediciones no invasivas, como las imágenes diagnósticas (2). La prevalencia reportada en algunos estudios osciló entre el $2.5 \%$ en el grupo de edad de $50-$ 59 años y el $14.5 \%$ en sujetos mayores de 70 años con un rango general de entre el 3\% al 10\% (3-5). Este síndrome puede variar clínicamente, y va desde la claudicación en los miembros inferiores, afectando principalmente los músculos de la pantorrilla, hasta la forma clásica determinada por la claudicación intermitente bilateral de los glúteos, la disfunción sexual, la reducción de los pulsos femorales, palidez y frialdad cutánea de los miembros inferiores $(4,6)$.

Se considera que la principal causa de esta patología es la producción de lesiones arterioscleróticas progresivas en la pared de la aorta abdominal con una trombosis superpuesta, especialmente en las personas con factores de riesgo como el tabaquismo, la hipertensión arterial (HTA), las dislipidemias, la obesidad y la diabetes mellitus tipo 2 (DM2), que aumentan el riesgo de padecer una enfermedad arterial periférica. Otro factor importante para tener en cuenta es la edad avanzada, con mayor frecuencia a partir de los sesenta años, principalmente en el sexo masculino $(4,5,7)$.

Al considerar los métodos de diagnóstico, las técnicas de imagen no invasivas se han convertido en los estudios preferidos para evaluar a los pacientes con sospecha de EOAI, ya que permiten analizar la anatomía vascular con gran precisión, especialmente cuando se utilizan medios de contraste que aportan datos como la perfusión u obstrucción del flujo sanguíneo $(6,8)$.
Esta patología reviste una importante causa de morbilidad y secuelas en los pacientes que la sufren, por lo que es evidente la necesidad de abordarla para divulgar la importancia de su sospecha. Un tratamiento oportuno traerá consigo un mejor resultado $\mathrm{y}$, teniendo en cuenta que el mecanismo por el que se desarrolla esta patología comparte etiología con otras enfermedades cardiovasculares, se hace imperativo resaltar la importancia del adecuado control lípido en los pacientes.

El objetivo de este manuscrito es presentar el caso clínico de un paciente con síndrome de Leriche cuyo diagnóstico trombótico fue confirmado por una angiotomografía computarizada con su respectiva reestructuración en 3D, pese a que la ecografía Doppler desestimó inicialmente la lesión trombótica. También se busca informar al lector sobre los datos propios de la patología, a través de una breve discusión de la literatura.

\section{Presentación de caso}

Paciente masculino de 71 años, con antecedentes de Enfermedad Pulmonar Obstructiva Crónica (EPOC) sin tratamiento, DM2 e HTA, quien ingresó al servicio de urgencias de una institución de Colombia, por dolor intenso y súbito en el miembro inferior izquierdo con 5 horas de evolución, asociado a parestesias, debilidad ipsilateral, diaforesis y frialdad distal. En el examen físico presentó ausencia de pulsos femorales, poplíteos y tibiales posteriores, además de cianosis distal en el miembro afectado. Se inició anticoagulación y analgesia con la posterior realización de una ecotomografía Doppler arterial en la que observó la oclusión de todo el miembro sin evidencia de proceso trombótico. Por las características del caso, se realizó una angiotomografía, observando trombo en la arteria ilíaca izquierda con oclusión total, procesando en tercera dimensión la respectiva imagen computarizada (Figura 1). Se llevó a cabo una trombo-embolectomía abierta con el fin de salvar la extremidad, debido a que la extensión de la lesión y su duración (más de cinco horas) así lo indicaban.

El paciente respondió adecuadamente con recuperación de la perfusión en la extremidad. Durante la hospitalización 
se realizó el manejo respectivo de comorbilidades y se buscaron posibles focos tromboembólicos con un ecocardiograma transtorácico y un Holter de ritmo por 24 horas, sin encontrar alteraciones como arritmia a nivel cardiaco ni coágulos alojados en la orejuela atrial. Se suspendió el anticoagulante cumarínico en su segundo día de tratamiento secundario a alta sensibilidad al encontrar niveles del International Normalized Ratio (INR) en 10.86, que se asocia a la aparición de equimosis alrededor de herida quirúrgica. El paciente evolucionó adecuadamente logrando normalidad en exámenes de laboratorio, en especial en el INR, y luego de iniciado el proceso de rehabilitación con fisioterapia se decidió dar egreso con control ambulatorio. Finalmente, se le solicitó al paciente la autorización para la publicación del caso.

Figura 1. Angiografía y reconstrucción que evidencian una obstrucción crítica a nivel de la arteria ilíaca izquierda.

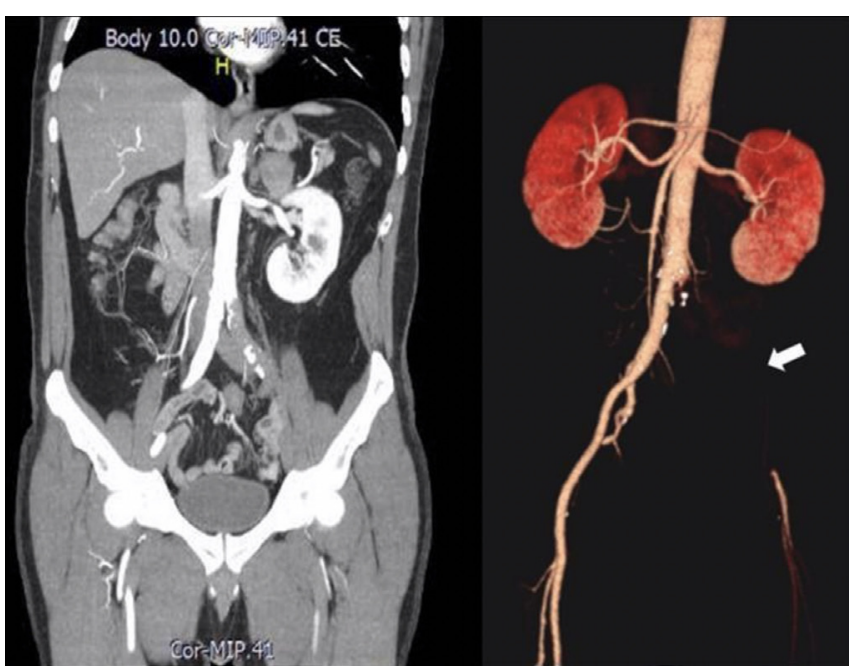

Fuente: Fotografías tomadas del historial clínico del paciente previo consentimiento informado.

\section{Discusión}

La claudicación intermitente es el principal síntoma en el síndrome de Leriche y está presente en el $5.5 \%$ de los pacientes diagnosticados con enfermedad arterial periférica reciente, y el $12.6 \%$ de los pacientes con un diagnóstico previo de la misma. Sin embargo, la medición de este síntoma no siempre determina la presencia o ausencia de la enfermedad $(4,5)$. Estas lesiones arterioscleróticas resultan de procesos patogénicos tales como el desplazamiento y la reducción de la matriz intracelular, la generación de depósitos minerales, inflamación crónica, neovascularización, ruptura de la superficie de la lesión lipídica y formación de trombo en el tejido fibromuscular $(9,10)$.

Para realizar el diagnóstico de esta enfermedad arterial periférica se utiliza principalmente un enfoque de estudio no invasivo. Inicialmente la historia clínica y los factores de riesgo del paciente deben orientar hacia la enfermedad. En el caso presentado, el dolor del miembro inferior izquierdo, parestesias, diaforesis, la cianosis y la ausencia de pulsos distales, así como la presencia de factores de riesgo (HTA y DM) fueron los que sugirieron el proceso isquémico asociado a un evento oclusivo del miembro inferior izquierdo (11). Las diferentes técnicas imagenológicas, además de participar en el establecimiento del diagnóstico, también influyen en la indicación del tratamiento quirúrgico o endovascular, debido a que estas técnicas permiten una visualización anatómica de los segmentos arteriales lesionados.

Uno de los métodos diagnósticos utilizados en el paciente fue el ultrasonido $(12,13)$. La ecografia Doppler arterial es una técnica que determina la hemodinamia, la localización y la extensión de las áreas arteriales ocluidas, pues evalúa en tiempo real la velocidad del flujo sanguíneo y la morfología de la curva espectral, permitiendo así reconocer las áreas afectadas hemodinámicamente (14). Pese a tener varias ventajas, como ser más económica, no invasiva y cómoda para el paciente, es una técnica que está indicada en su mayoría para patologías venosas. En procesos arteriales puede ser menor su índice de predicción diagnóstica, por lo que se hacen necesarias imágenes más sensibles y específicas, como con la angiotomografia, que ofrece una visión más compleja del trayecto arterial en conjunto (12-14).

La angiotomografía o angiografía por tomografía computarizada, también es una técnica no invasiva con alta sensibilidad (del 95\% al 96\%) y especificidad (del 96\% al 98\%) para detectar estenosis aorto-ilíaca. Esta se ha convertido en una gran herramienta diagnóstica, debido a los grandes alcances imagenológicos como la obtención de imágenes iso volumétricas que permiten evaluar todo el trayecto arterial en los diferentes planos (axial, sagital y coronal, en este caso) por medio del uso de ciertas dosis de medios de contraste (yodado) que permiten dibujar las áreas arteriales que se requiere, combinando el uso de rayos $\mathrm{X}$ y el análisis computarizado de las imágenes (15). Esta técnica permite visualizar áreas arteriales calcificadas, así como posibles colaterales resultantes del proceso oclusivo, incluso en pacientes sin claudicación $(1,3)$. La angiotomografía ha desplazado a la angiografía convencional, ya que esta presenta mayores efectos adversos debido al uso de mayores dosis de contraste intravenoso, particularmente en pacientes con 
insuficiencia renal. La angiografía convencional también tiene el riesgo de generar reacciones alérgicas $(14,15)$. Por esto, en la actualidad, la angiotomografía es una de las técnicas preferidas y más convenientes frente a la sospecha de procesos oclusivos. Además, la imagen de corte transversal tiene la ventaja de delinear la patología no vascular; una ventaja potencialmente vital, reconociendo que esta enfermedad se ha asociado a comorbilidades de alto riesgo $(12,15,16)$.

Considerando el tratamiento, el reparo quirúrgico, tanto el injerto bypass como la endarterectomía han demostrado una adecuada efectividad y alivio de síntomas además de proveer una buena permeabilidad a largo plazo (4). Las técnicas endovasculares han demostrado ser una alternativa mínimamente invasiva en el tratamiento del síndrome de Leriche con una reducción de la morbilidad comparada con la reparación quirúrgica en relación con la estancia hospitalaria y sus complicaciones. Sin embargo, en situaciones de gran extensión o gran compromiso del estado vascular, como en el paciente acá referido, la técnica endovascular no se ha recomendado como estrategia primaria frente a la técnica quirúrgica, pues se ha reconocido superioridad en términos de permeabilidad y durabilidad de la reperfusión. Al final, la elección del tratamiento también está afianzada a las capacidades propias de la institución y, en algunos casos, el manejo híbrido puede ser una opción, teniendo en cuenta que estos pacientes deben tener un seguimiento y un control de comorbilidades de base y de su estilo de vida $(4,17-20)$.

\section{Conclusiones}

El avance imagenológico de los últimos años ha permitido proceder en patologías arteriales de manera menos invasiva, situación que antes era impensable. La angiografía por tomografía computarizada es una técnica no invasiva con alta sensibilidad y especificidad para detectar estenosis aorto-ilíaca y se ha convertido en una gran herramienta diagnóstica por sus grandes alcances imagenológicos, que permiten evaluar todo el trayecto arterial en los diferentes planos, gracias al uso de medios de contraste, superando en utilidad a una ecografía y permitiendo una amplia ayuda en comorbilidades de base, ampliando los hallazgos y detalles posibles.

\section{Conflicto de intereses.}

Los autores declaran no tener conflicto de intereses.

\section{Referencias}

1. Keller K., Beule J., Oliver Balzer J., Coldewey M., Munzel T., Dippold W., et al. A 56-year-old man with co-prevalence of Leriche syndrome and dilated cardiomyopathy: case report and review. Wien Klin Wochenschr. 2013;126(5-6):163-8. Doi: https://doi. org/10.1007/s00508-013-0476-2

2. Brown K., Gonzalez L. Leriche Syndrome. StatPearls [Internet]. 2019; (consultado el 13 noviembre del 2019). Disponible en: https://www.ncbi.nlm.nih.gov/ books/NBK538248/

3. Morotti A., Busso M., Cinardo P., Bonomo K., Angelino V., Cardinale L., et al. When collateral vessels matter: asymptomatic Leriche syndrome. Clin Case Reports. 2015;3(11):960-1. doi:10.1002/ ccr3.390

4. Fernández M. R., Luján V. P., Martínez J, Díaz J. J., RamírezE.Enfermedadoclusiva aortoilíaca osíndrome de Leriche. Rev Colomb Cirugía. 2018;32(3):214-22. Doi: https://doi.org/10.30944/20117582.27

5. Norgren L., Hiatt W. R., Dormandy J. A., Nehler M. R., Harris K. A., Fowkes F.G., et al. Inter-society consensus for the management of peripheral arterial disease (TASC II). Int Angiol. 2007;26(2):81-157. Doi: https://doi.org/10.1016/j.jvs.2006.12.037

6. Ahmed S., Raman S. P., Fishman E. K. CT angiography and 3D imaging in aortoiliac occlusive disease: collateral pathways in Leriche syndrome. Abdom Radiol. 2017;42(9):2346-57. Doi: https://doi. org/10.1007/s00261-017-1137-0

7. Oviedo Gamboa I., Herbas Bernal R., Zegarra Santiesteban W. Diagnóstico por tomografía del Síndrome de Leriche: reporte de un caso clínico. Gac Med Bol. 2013;36(2):105-7. Disponible en: http://www.scielo.org.bo/scielo.php? script $=$ sci arttext\&pid=S1012-29662013000200012\&lng=es.

8. Met R., Bipat S., Legemate D. A., Reekers J. A., Koelemay M. J. W. Diagnostic Performance of Computed Tomography Angiography in Peripheral Arterial Disease. JAMA. 2009;301(4):415-24. doi: 10.1001/jama.301.4.415

9. Florenzano F. Fisiopatología de la placa ateroesclerótica. Rev méd Clín Las Condes. 2000;11(2):1-11.

10. Bertomeu Ruiz A., Zambón Rados D. La placa aterogénica: fisiopatología y consecuencias clínicas. Med Integr [Internet]. 2002;40(9):394 405. Disponible en: http://www.elsevier.es/es-revista-medicina-integral63-articulo-la-placa-aterogenica-fisiopatologiaconsecuencias-13041112

11. Spittell J. A. Diagnosis and management of occlusive peripheral arterial disease. Curr ProblCardiol. 1990;15(1). Doi: $\underline{\text { https://doi.org/10.1016/0146-2806(90)90024-k }}$ 
12. SerranoHernandoF.J., MartínConejeroA.Enfermedad arterial periférica: aspectos fisiopatológicos, clínicos y terapéuticos. Rev Española Cardiol [Internet]. 2007;60(9):969-82. Disponible en: https://linkinghub. elsevier.com/retrieve/pii/S0300893207751473

13. Jones D.W., Schwartz G.L., Safian R. D., Hirsch A. T., Spittell J. A., Easton J. D., et al. Atherosclerotic Vascular Disease Conference. Circulation. 2004;109(21):2634 42. Doi: https://doi.org/10.1161/01. CIR.0000128522.98016.19

14. Selfa Moreno S. La ecografía Doppler en el diagnóstico de la isquemia crónica de MMII. Hosp Lluis Alcanyis [Internet]. 2014. Disponible en: http:// www.srcv.org/repo/static/public/jornadasDoppler/08Doppler_Arterial.pdf

15. Sociedad D., Cardiología E De, Accidentes E De, European C, Organisation S, Michel DT, et al. Guía de práctica clínica de la ESC sobre diagnóstico y tratamiento de las enfermedades arteriales periféricas. Rev Esp Cardiol. 2012;65(2). Disponible en: https:// www.revespcardiol.org/contenidos/static/avance guias/Guia2012_2.pdf

16. Haberman D., Froullet C., Bassuk A., Martínez A., BeresñakA., GurfinkelE. Angiotomografía computada multicorte 64 en patología de la arteria aorta. Rev argent radiol. 2008;72(3):277-86. Disponible en: http://www.scielo.org.ar/scielo.php?script=sci arttext\&pid=S1852-99922008000300004\&lng=es.

17. Bae M., Chung S. W., Lee C.W., Huh U., Kim M. S., Song S. H. Risk factors associated with postoperative prosthetic graft patency in Leriche syndrome. Asian J Surg [Internet]. 2019;42(1):235-9. Disponible en: https://doi.org/10.1016/j.asjsur.2018.08.009

18. Leville C. D., Kashyap V. S., Clair D. G., Bena J. F., Lyden S. P., Greenberg R. K., et al. Endovascular management of iliac artery occlusions: Extending treatment to TransAtlantic Inter-Society Consensus class C and D patients. J Vasc Surg. 2006;43(1):32-9. Doi: https://doi.org/10.1016/j.jvs.2005.09.034

19. Jongkind V., Akkersdijk G. J. M., Yeung K. K., Wisselink W. A systematic review of endovascular treatment of extensive aortoiliac occlusive disease. J Vasc Surg [Internet]. 2010;52(5):1376-83. Disponible en: http://dx.doi.org/10.1016/j.jvs.2010.04.080

20. Indes J. E., Pfaff M. J., Farrokhyar F., Brown H., Hashim P., Cheung K., et al. Clinical Outcomes of 5358 Patients Undergoing Direct Open Bypass or Endovascular Treatment for Aortoiliac Occlusive Disease: A Systematic Review and Meta-Analysis. J Endovasc Ther. 2013;20(4):443-55. Doi: https://doi. org/10.1583/13-4242.1 